\title{
A new kind of big science
}

The 5 years since the first detection of gravitational waves have witnessed the rise of multimessenger astronomy, a field that expands our understanding of astrophysical processes and reshapes the way science is done.

On 14 September 2015, gravitational waves from a black hole merger hundreds of megaparsecs away rippled through the Earth. An unremarkable event on astrophysical scales, it marked a spectacular achievement for humankind because for the first time, gravitational waves were detected. Over the past 5 years, detecting gravitational waves has become almost routine, with a public alert system in place. These advances have affected not only scientific understanding, but the very way science is carried out.

Multi-messenger astrophysics is a new field that uses information carried by photons, cosmic rays, neutrinos and gravitational waves to observe and understand violent astrophysical phenomena such as neutron star mergers or blazars. In 2017, two such events were recorded: GW170817, a neutron star merger releasing gravitational waves and electromagnetic radiation, and the detection of high-energy neutrinos from TXS $0506+056$, a very high-energy blazar emitting across the electromagnetic spectrum. These events (known as transients) are ephemeral and to observe them many instruments have to be ready, and their teams must be able to react quickly.

Multi-messenger observation campaigns are different from other big science enterprises. Big surveys, such as the Sloan Digital Sky Survey or the Dark Energy Survey, are carefully planned, instrument time is scheduled and then regions of the sky are browsed for long periods of time. Data are processed, stored and prepared for regular data releases. Collider experiments, such as ATLAS and CMS, or accelerator-based neutrino experiments, such as T2K, are especially designed for specific purposes with control over the characteristics of the events and the detectors and data acquisition optimized to get out the most information. These are in stark contrast to a fast-paced, multi-instrument observation campaign, which is a race against time to get the most out of the limited observation window using different types of independently operated instruments in various locations around the globe, which may or may not be optimized for that particular type of observation. This unusual situation poses unique challenges that have been recently discussed ${ }^{1}$.

The timely notification of newly discovered events is a key issue for multi-messenger astrophysics, requiring a reliable system of alerts and fast communication and coordination. Data availability and accessibility are also other important factors, in particular in the case of time-critical observation campaigns. However, data should be not only available, but FAIR (findable, accessible, interoperable and reusable), which is particularly challenging for large volumes of data coming from different instruments and covering a wide range of wavelengths, time scales and multiple messengers ${ }^{1}$. These needs call for better instrument coordination and more international funding and collaboration.

In this issue, we explore examples of how the astrophysics community has been reorganizing itself to respond to these challenges. There are several networks of instruments working together to enhance the potential for new discoveries, such as AMON (see Comment by Miguel Mostafa), GROWTH (see Comment by Mansi Kasliwal) or SNEWS (see Comment by Alec Habig and Kate Scholberg). These networks require the development of complex cyberinfrastructure and the use of AI technologies for data mining. In the past 2 years, new collaborations have been formed, such as GRANDMA (see Comment by Sarah Antier) and ENGRAVE (see Comment by Andrew Levan and Peter Jonker), that combine in their organization practices from both particle physics and astronomy trying to harness an array of heterogeneous resources, including citizen science in the case of GRANDMA. For parts of the astrophysics community such large and diverse collaborations are "a new experience", say Levan and Jonker.

These are exciting times. Never before have scientists needed a specialized technological and human infrastructure to alert and operate a global network of instruments to be able to squeeze the most information out of unpredictable transient events. Never before has science required such a level of international coordination and collaboration. In the coming years we should be expecting not only amazing discoveries, but also further sociological and technological changes reflecting these unprecedented needs.

- Bradley Cenko, S. et al. International coordination of multi-messenger transient observations in the 2020 s and beyond: Kavli-IAU White Paper. Preprint at arXiv:2007.05546 (2020). 